\title{
Amyloidose in der Pneumologie
}

\section{Amyloidosis in Pneumology}

Autoren

Institute

\section{Hagmeyer ${ }^{1}$, S. Stieglitz ${ }^{1}$, C. Röcken², W. Randerath ${ }^{1}$}

${ }^{1}$ Krankenhaus Bethanien gGmbH, Klinik für Pneumologie und Allergologie, Zentrum für Schlaf- und Beatmungsmedizin, Solingen

${ }^{2}$ Institut für Pathologie, Christian-Albrechts-Universität, Kiel eingereicht 31.1 .2012 akzeptiert nach Revision 18. 4. 2012

\section{Bibliografie}

Dol http://dx.doi.org/ 10.1055/s-0032-1309811 Online-Publikation: 12.6.2012 Pneumologie 2012; 66: 483-492 (c) Georg Thieme Verlag KG Stuttgart · New York ISSN 0934-8387

\section{Korrespondenzadresse} Dr. med. Lars Hagmeyer Bethanien Krankenhaus Solingen

Aufderhöher Straße 169-175

42699 Solingen

lars.hagmeyer@klinik-

bethanien.de

\section{Zusammenfassung \\ $\nabla$}

Im pneumologischen Fachgebiet ist die Amyloidose eine seltene Krankheitsentität. Wahrscheinlich gibt es jedoch eine relevante Dunkelziffer an nicht diagnostizierten Fällen. Nach wie vor wird diese Erkrankung kaum ins differenzialdiagnostische Kalkül bei der Abklärung unklarer respiratorischer Beschwerden und bildmorphologischer Befunde mit einbezogen. Vielmehr handelt es sich bei den meisten Fällen um Zufallsdiagnosen, in der Regel erfolgt die Diagnosestellung erst durch den Pathologen anhand einer Gewebeprobe. Nur wenn die mannigfaltigen Manifestationsformen dem Kliniker bekannt sind, wird die Amyloidose im Einzelfall in der Differenzialdiagnose Berücksichtigung finden. Bei Verdacht auf das Vorliegen einer Amyloidose ist der zielführende diagnostische Schritt die Durchführung einer Gewebebiopsie. Wird die Diagnose in der Kongorotfärbung bestätigt, ist eine Subtypisierung des Amyloidproteins erforderlich, um nach möglichen behandelbaren Grunderkrankungen suchen zu können und die Therapie zu planen. Die Klassifikation der Amyloidosen erfolgt anhand des detektierten Amyloidproteins, klinisch werden hereditäre von erworbenen und lokale von generalisierten Formen abgegrenzt. Neben der Behandlung einer eventuell vorliegenden Grunderkrankung stehen für die generalisierten Amyloidosen systemische Therapien zur Verfügung. Lokale Amyloidosemanifestationen können oft gut mit spezifischen Lokalmaßnahmen behandelt werden. In dieser Übersichtsarbeit soll ein Überblick über die Pathophysiologie, die Klassifikation der Amyloidose-Unterformen, sowie deren Diagnostik und Therapie ermöglicht werden. Ein spezielles Augenmerk soll dabei auf die besonderen Manifestationen aus dem pneumologischen Fachgebiet und deren spezifische Therapiemöglichkeiten gelenkt werden.

\section{Abstract \\ $\nabla$}

Amyloidosis is a rare disorder within the field of pneumology, however, it is estimated that there are a relevant number of unreported cases. In routine clinical practice, the disease is seldom considered in the diagnostic work-up of unclear respiratory symptoms and radiological findings. For amyloidosis to be considered as a differential diagnosis, the clinician must be aware of the broad variety of its clinical manifestations. In cases where amyloidosis is suspected, it is important to obtain an early tissue biopsy. If the diagnosis can be confirmed by Congo red staining, further subtyping of the amyloid protein is necessary in order to identify a treatable cause of the disease. The amyloidoses are classified according to the type of amyloid protein, with clinical subclassifications distinguishing hereditary from acquired forms and localised from generalised manifestations. Apart from causal therapy of the primary disease, the treatment of generalised amyloidosis includes specific systemic therapy. The majority of localised forms are treated with specific local interventions showing good longterm results. This review outlines the pathophysiology, classification, diagnostic pathways and therapeutic modalities in amyloidosis. Furthermore, typical manifestations of amyloidosis of the lung and the specific treatment options are discussed. 


\section{Amyloid}

Rudolph Virchow beschrieb im Jahr 1854 erstmals beim Menschen eine makroskopische Gewebsveränderung, die ein charakteristisches Färbeverhalten bei der Blaufärbung mit gelöstem Jod (Lugolsche Lösung) zeigte. Diese Anfärbbarkeit mit Jod war „stärkeähnlich“, Virchow wählte die deskriptive Bezeichnung „Amyloid“ (griechisch: ó $\mu$ $\lambda_{0}=$ Stärke).

Heute nutzt der Pathologe als histologische Nachweismethode für Amyloid die Kongorot-Färbung in Kombination mit der Polarisationsmikroskopie. Nach der Farbstoffbindung an das Amyloid zeigt sich polarisationsmikroskopisch eine charakteristische anomale Polarisationsfarbe ( $\bullet$ Abb.1). In der Hämatoxylin-EosinFärbung färbt sich Amyloid homogen eosinrot an ( $\bullet$ Abb. 1). Die klare Abgrenzung der Substanz gegenüber den verschiedenen Hyalinarten gelang elektronenmikroskopisch erstmals im Jahr 1959 durch den Nachweis der pathognomonischen starren, nichtverzweigten Fibrillen variabler Länge mit einem mittleren Durchmesser von 7-10 nm [1].

Bei den Amyloidfibrillen handelt es sich um Polypeptidaggregate mit einer Cross- $\beta$-Struktur. Die Cross- $\beta$-Struktur stellt das gemeinsame Bauprinzip aller Amyloidfibrillen dar. Die inzwischen über 25 verschiedenen Amyloidproteine leiten sich ausnahmslos von autologen, physiologischen Vorläuferproteinen ab. Die an sich löslichen Vorläuferproteine ändern im Verlauf der Amyloid- bildung oft ihre Tertiärstruktur, aggregieren dann miteinander und lagern sich als unlösliche Fibrillen extrazellulär, teilweise auch intrazellulär, systemisch oder lokalisiert ab [2, 3]. Die einzelnen Amyloidarten werden anhand der unterschiedlichen Proteinkomponenten der Fibrillen voneinander unterschieden (๑ Tab. 1).

\section{Amyloidose}

$\nabla$

Die Amyloidose wird heute als Erkrankung definiert, die aus der extrazellulären, seltener intrazellulären, Ablagerung von Amyloid in parenchymatösen Organen, Gefäßen oder Nerven resultiert. Der Nachweis einer Amyloidose erfolgt histologisch durch die Biopsie eines betroffenen Organs oder bei den systemischen Amyloidosen weniger invasiv beispielsweise durch Aspiration von subkutanem Fettgewebe [4]. Biopsien aus abdominellem Fettgewebe, Lippenschleimhaut oder rektaler Schleimhaut zeigten in Studien eine Sensitivität von bis zu $80 \%$, enttäuschten allerdings in der klinischen Routine durch falsch negative Ergebnisse in bis zu 50\% der Amyloidosepatienten [5 - 7]. Zur näheren Charakterisierung des Amyloids können die oben beschriebenen feingeweblichen Untersuchungen um immunhistochemische Färbungen, die Immunogold-Elektronenmikroskopie und Techniken der Sequenzierung der Amyloidproteine erweitert werden.
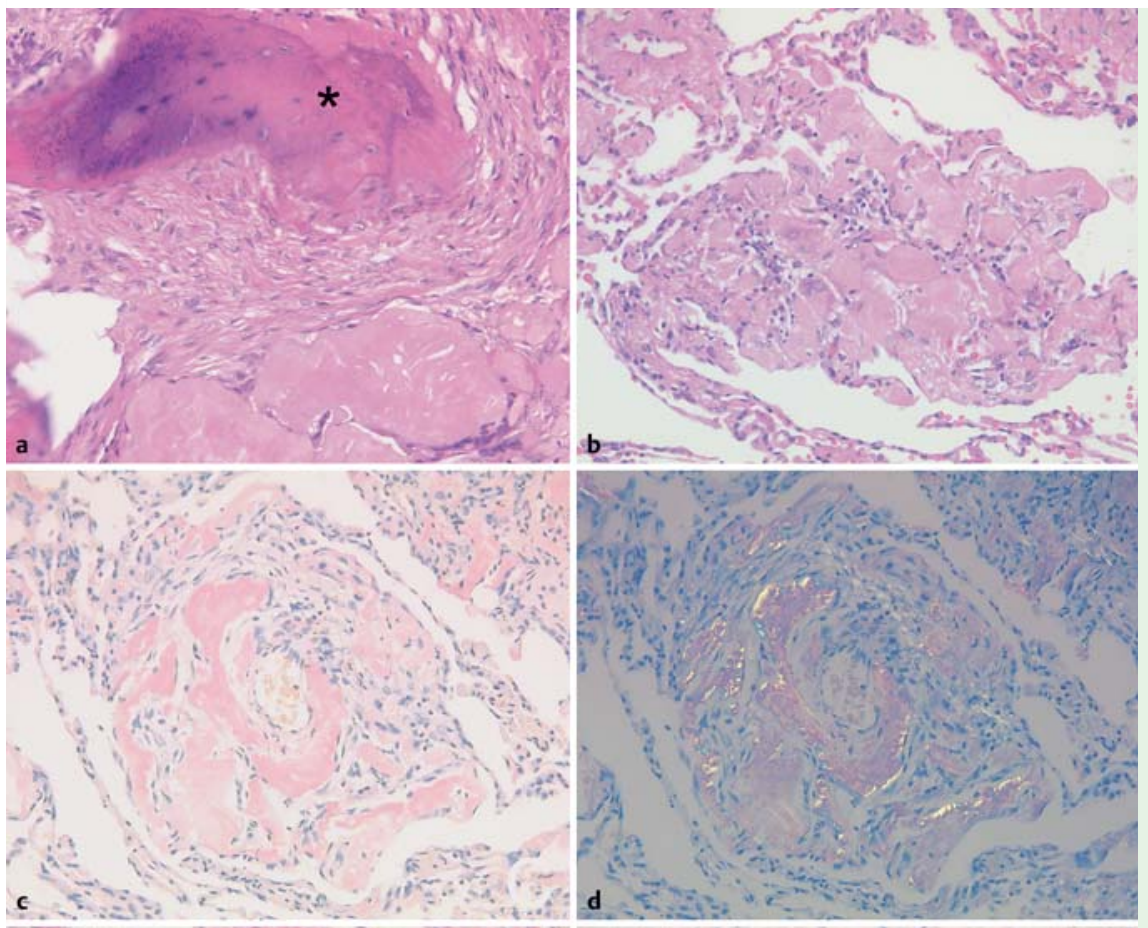

Abb. 1 Pulmonale AL-Amyloidose- $\lambda$-Leichtkette bei einem 67 Jahre alten Mann: Im HämatoxylinEosin-gefärbten Schnittpräparat finden sich Ablagerungen eines homogen-eosinroten Materials $(\mathbf{a}, \mathbf{b})$, das teilweise tumorförmig imponiert (a), teilweise aber auch vaskulär und alveolär-septal abgelagert ist (b). Die Amyloidablagerungen enthalten heterotope Ossifikationen (a, Sternchen). In der Kongorotfärbung findet sich polarisationsoptisch eine charakteristische anomale Polarisationsfarbe (c, d). Die immunhistologische Klassifikation zeigt eine kräftige homogene Immunreaktion mit einem gegen $\lambda$-Leichtkette gerichteten Antikörper (e), wohingegen sich die Amyloidablagerungen mit einem gegen AA-Amyloid gerichteten Antikörper immunnegativ verhalten (f). Hämatoxylin und Eosin $(\mathbf{a}, \mathbf{b})$, Kongorotfärbung im Durchlicht (c) und im polarisierten Licht (d). Anti- $\lambda$-Leichtkette-Antikörper (e), anti-AA-Amyloid-Antikörper (f). Originalvergrößerungen $\times 200$.

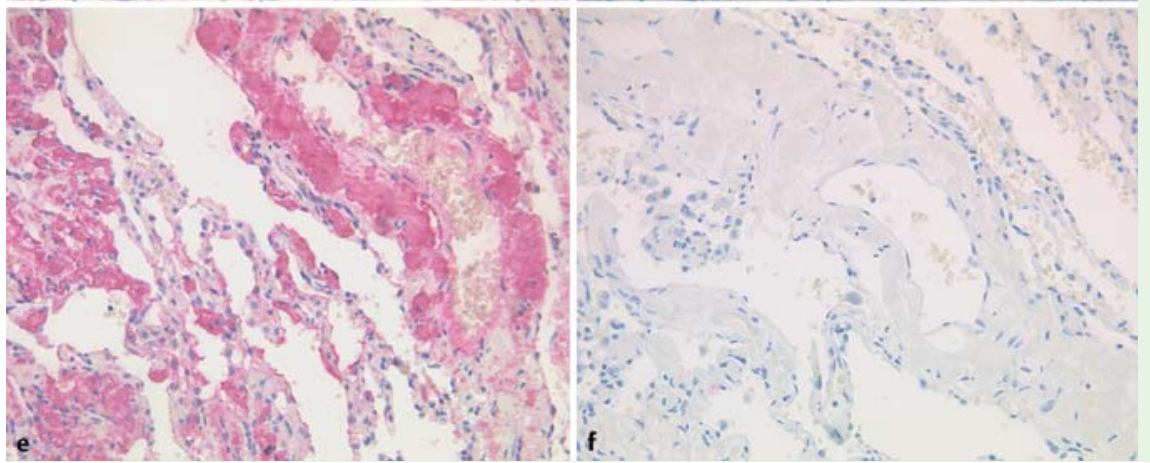


Tab. 1 Nomenklatur der Amyloidosen.

\begin{tabular}{|c|c|c|c|c|}
\hline Amyloidprotein & Vorläuferprotein & $\begin{array}{l}\text { Systemisch (S) } \\
\text { oder lokal (L) }\end{array}$ & $\begin{array}{l}\text { Hereditär }(H) \text { oder } \\
\text { erworben }(E)\end{array}$ & Amyloidose/Grunderkrankung \\
\hline $\mathrm{AL}$ & Immunglobulin-Leichtkette & $S, L$ & $\mathrm{E}$ & Multiples Myelom, primäre AL-Amyloidose \\
\hline $\mathrm{AH}$ & Immunglobulin-Schwerkette & $S, L$ & $\mathrm{E}$ & Multiples Myelom, primäre AH-Amyloidose \\
\hline ATTR & Transthyretin & $S, L ?$ & $\mathrm{H},(\mathrm{E} ?)$ & $\begin{array}{l}\text { Familiäre Amyloidpolyneuropathie (portugiesischer } \\
\text { Typ), senile kardiovaskuläre Amyloidose, senile } \\
\text { pulmonale Amyloidose }\end{array}$ \\
\hline $\mathrm{A} ß 2 \mathrm{M}$ & B2-Mikroglobulin & $S, L ?$ & $\mathrm{E}$ & Chronische Hämodialyse, Gelenke \\
\hline $\mathrm{AA}$ & Serum-Amyloid A & S & $\mathrm{E}$ & sekundär, reaktiv \\
\hline AApoAl & Apolipoprotein A-I & $S, L$ & $\mathrm{H}, \mathrm{E}$ & Hereditäre systemische Amyloidose, Atherosklerose \\
\hline AApoAll & Apolipoprotein A-II & S & $\mathrm{H}$ & Hereditäre renale Amyloidose \\
\hline AGel & Gelsolin & S & $\mathrm{H}$ & $\begin{array}{l}\text { Familiäre Amyloidpolyneuropathie (finnischer Typ), } \\
\text { gittrige Hornhautdystrophie }\end{array}$ \\
\hline Alys & Lysozym & S & $\mathrm{H}$ & Familiäre viszerale Amyloidose \\
\hline AFib & Fibrinogen alpha-Kette & S & $\mathrm{H}$ & Hereditäre systemische Amyloidose \\
\hline ACys & Cystatin C & S & $\mathrm{H}$ & Familiäre Amyloidose (Island-Typ) \\
\hline ABri & ABriPP & S & $\mathrm{H}$ & Familiäre Demenz (britischer Typ) \\
\hline ADan & ADanPP & L & $\mathrm{H}$ & $\begin{array}{l}\text { Heredopathia ophthalmo-oto-encephalica } \\
\text { (dänischer Typ) }\end{array}$ \\
\hline$A B$ & AßPP & L & $\mathrm{E}, \mathrm{H}$ ? & $\begin{array}{l}\text { Senile Demenz vom Alzheimer-Typ, Down-Syndrom, } \\
\text { Amyloidangiopathie }\end{array}$ \\
\hline APrP & Prion-Protein & $\mathrm{L}$ & $\mathrm{E}$ & Spongiforme Enzephalopathie \\
\hline ACal & (Pro)Calcitonin & L & $\mathrm{E}$ & Medulläres Schilddrüsenkarzinom \\
\hline AIAPP & Inselamyloid-Polypeptid & $\mathrm{L}$ & $\mathrm{E}$ & Langerhans-Inseln (Diabetes mellitus), Insulinom \\
\hline AANF & Atrionatriuretisches Peptid & $\mathrm{L}$ & E & Herzvorhof, Vorhofflimmern \\
\hline APro & Prolaktin & $\mathrm{L}$ & E & Hypophyse, Hypophysenadenom \\
\hline Alns & Insulin & $\mathrm{L}$ & $\mathrm{E}$ & iatrogen \\
\hline AMed & Lactatherin & L & $\mathrm{E}$ & Amyloid der Aortenmedia \\
\hline AKer & Keratoepithelin & $\mathrm{L}$ & $\mathrm{E},(\mathrm{H}$ ?) & Cornea, gittrige Hornhautdystrophie \\
\hline APin & FLJ20513-assoziiertes Protein & $\mathrm{L}$ & $\mathrm{E}$ & Pindborg-Tumor \\
\hline ALac & Lactoferrin & L & E & Korneales Amyloid (Trichiasis) \\
\hline ASeg & Semenogilin I & L & E & Samenblase \\
\hline ATau & Tau-Protein & $\mathrm{L}$ & $\mathrm{E}$ & Hirn \\
\hline
\end{tabular}

Modifiziert nach den Empfehlungen des Nomenclature Committee of the International Society of Amyloidosis [9].

Sehr verlässliche Informationen liefert das kosten- und arbeitsintensive Verfahren der Laser-Mikrodissektion mit Massenspektrometrie (LMD/MS) [8]. Zur Erfassung insbesondere der angeborenen Formen sind anschließend Analysen genomischer DNA hilfreich [6].

Die systematische Klassifikation der Amyloidoseformen in den geltenden Leitlinien berücksichtigt einerseits, ob eine erworbene oder eine hereditäre Form der Erkrankung vorliegt. Andererseits erfolgt eine Einteilung nach dem Amyloidprotein $[9,10]$. In - Tab. 1 werden die bekannten Amyloidoseformen systematisch zusammengefasst.

Mit diesem Ansatz wird der Pathogenese und damit den wesentlichen Unterschieden bezüglich der Prognose und der Therapieoptionen Rechnung getragen. Je nach Amyloidoseform und Stadium der Erkrankung reicht das therapeutische Spektrum von lokalen Behandlungsmaßnahmen über medikamentöse antiinflammatorische Konzepte oder Chemotherapie-Schemata bis hin zur Organtransplantation [10]. Das Konzept, allein die primären, meist lokalen Amyloidoseformen von sekundären, in der Regel generalisierten (Begleit-)Amyloidosen abzugrenzen, ist heute weitgehend verlassen. Dennoch sollen an dieser Stelle häufige isolierte Organamyloidosen Erwähnung finden, die klassische klinische Krankheitsbilder verursachen. Zu diesen Formen zählen z.B. die zerebrale Amyloidose beim M. Alzheimer, die mit Vorhofflimmern assoziierte Herzvorhofamyloidose und die mit
Diabetes mellitus vergesellschaftete Inselamyloidose des Pankreas [10].

Bei den hereditären Formen der Amyloidose führen Keimbahnmutationen zu einer Veränderung in der Primärstruktur eines Proteins, das dadurch eine deutlich gesteigerte Neigung zur Polypeptid-Aggregation und Bildung von Amyloid aufweist. In den vergangenen Jahren wurden zahlreiche hereditäre Amyloidosen beschrieben, bei denen das Vorläuferprotein identifiziert wurde. Die häufigste in Deutschland vorkommende hereditäre Amyloidose wird durch Transthyretin(TTR)-Varianten verursacht. Klinisch imponiert dabei zum Beispiel das Bild einer familiären Amyloid-Polyneuropathie (FAP) oder familiären Kardiomyopathie (FAC). Untersuchungen haben gezeigt, dass das Durchschnittsalter der Patienten mit hereditärer ATTR-Amyloidose zum Diagnosezeitpunkt 62 Jahre beträgt [11] und deshalb als solche oft nicht erkannt wird. Im November 2011 hat die Europäische Arzneimittelbehörde (EMA) Tafamidis (Vyndaquel ${ }^{\circledR}$ ) zur Behandlung der familiären Amyloid-Polyneuropathie vom Transthyretin-Typ (TTR-FAP) bei erwachsenen Patienten mit symptomatischer Polyneuropathie im Stadium 1 zugelassen.

Der überwiegende Anteil der erworbenen Amyloidosen entsteht auf dem Boden chronisch entzündlicher Prozesse, monoklonaler Gammopathien oder bei Langzeitdialysepatienten. 


\section{AL-Amyloidosen}

Die AL-Amyloidose ist ein heterogenes Krankheitsbild. Bis auf wenige Ausnahmen entsteht die Erkrankung im Rahmen einer monoklonalen Plasmazellerkrankung. Diese kann sich als nichtmaligne monoklonale Gammopathie unklarer Signifikanz (MGUS), als Immunozytom oder als malignes Plasmozytom bzw. multiples Myelom präsentieren. Im Rahmen der Erkrankung synthetisierte Leichtketten oder Leichtkettenfragmente finden sich zu Amyloidfibrillen zusammengelagert im Gewebe. Bis zu vier Prozent der Patienten mit einem MGUS entwickeln im Durchschnitt innerhalb von neun Jahren nach Diagnosestellung eine AL-Amyloidose $[2,3,12]$. Ungefähr $10 \%$ aller Patienten mit einem multiplen Myelom leiden gleichzeitig an einer AL-Amyloidose [10].

Die Diagnose einer monoklonalen Plasmazellerkrankung erfolgt mittels Elektrophorese und Immunfixation aus Urin oder Serum, quantitativer Bestimmung von Leichtketten im Serum sowie Nachweis einer klonalen Plasmazellvermehrung im Knochenmark. Klinisch kommt es typischerweise zu Amyloid-Ablagerungen in viszeralen Organen (Niere, Herz, Magen-Darm-Trakt, Leber, Milz, Lunge), in der Zunge und in peripheren Nerven oder Blutgefäßen. Die Risikostratifizierung erfolgt unter Berücksichtigung der Organbeteiligung, insbesondere eine Herzbeteiligung ist häufig prognosebestimmend. In den seltenen Fällen, in denen bei einer AL-Amyloidose keine monoklonale Plasmazellerkrankung gefunden wird, imponiert primär häufig das Bild einer lokalen Organamyloidose. Dennoch wird auch bei diesen Patienten das Vorliegen einer monoklonalen Plasmazellerkrankung diskutiert, die sich (noch) nicht systemisch manifestiert. Im Jahr 2004 wurden vom United Kingdom Myeloma Forum Leitlinien veröffentlicht, die ein klar standardisiertes Vorgehen bei der Erstabklärung und der Verlaufsevaluation einer AL-Amyloidose empfehlen ( $\bullet$ Tab. 2).

Eine spezifische Amyloidose-Therapie steht bis heute nicht zur Verfügung. Die Behandlung der systemischen AL-Amyloidose zielt auf eine Suppression der zugrundeliegenden PlasmazellDyskrasie ab und wird flankiert von supportiven Maßnahmen zum Erhalt der jeweils beeinträchtigten Organfunktion. Die spezifische Behandlung orientiert sich an den Leitlinien für das multiple Myelom. Einen umfassenden und differenzierten Überblick über die bei generalisierten AL-Amyloidosen zur Verfügung stehenden medikamentösen Therapiekonzepte und deren Indikationsstellung bieten die einschlägigen Leitlinienempfehlungen der britischen und der deutschen Amyloidosegesellschaft $[6,10]$. Neuere Arbeiten konnten zeigen, dass sich mit einer Kombinationstherapie unter Einschluss neuerer Substanzen wie Bortezomib gute Remissionsdaten erzielen lassen $[13,14]$. Einen Überblick über die zur Verfügung stehenden Therapieoptionen und Indikationen bietet $\bigcirc$ Tab. 3 .

Nicht alle AL-Amyloidosen manifestieren sich generalisiert. Sofern lediglich eine lokale Form vorliegt, sollte keine Behandlung mit einer Chemotherapie erfolgen. Zu beachten ist in diesem Zusammenhang, dass Nicht-AL-Amyloidosen, also insbesondere die sekundären, hereditären und dialyseassoziierten Formen, kein Ansprechen auf eine Chemotherapie zeigen.

\section{AA-Amyloidosen}

Beim familiären Mittelmeerfieber, bei chronischen Entzündungen (z. B. Tuberkulose, Bronchiektasen, Sarkoidose, Osteomyelitis, chronisch entzündliche Darmerkrankungen, rheumatologische Erkrankungen etc.), bei der Castleman-Krankheit und bei Malignomen kann es zu einer vermehrten Bildung des Vorläuferproteins Serum-Amyloid A kommen, einem Akutphase-Protein $[10,15-17]$. Bei langjährigem chronischem Krankheitsverlauf kommt es zur vaskulären und interstitiellen Ablagerung als AAAmyloid in verschiedenen Organen. Typischerweise kommt es bei dieser Form zu Organmanifestationen im Bereich der Nieren, der Leber und der Milz. Regelhaft wird eine Darmbeteiligung beobachtet. Die Therapie basiert im Wesentlichen auf einer Behandlung der ursächlichen Grunderkrankung. So kann bei den AA-Amyloidosen auf dem Boden von periodischen Fiebersyndromen (v.a. familiäres Mittelmeerfieber) eine Therapie mit Colchicin versucht werden.

Tab. 2 Diagnostisches Vorgehen bei vermuteter AL-Amyloidose. Modifiziert nach: Guidelines on the diagnosis and management of AL-Amyloidosis [6].

\begin{tabular}{|c|c|c|c|c|c|}
\hline & $\begin{array}{l}\text { Nachweis von } \\
\text { Amyloid }\end{array}$ & $\begin{array}{l}\text { Bestimmung des } \\
\text { Amyloidtyps }\end{array}$ & $\begin{array}{l}\text { Evaluierung der } \\
\text { Organbeteiligung }\end{array}$ & $\begin{array}{l}\text { Untersuchung der } \\
\text { zugrundeliegenden } \\
\text { Plasmazell-Dyskrasie }\end{array}$ & Monitoring \\
\hline Pathologie & $\begin{array}{l}\text { Biopsie und Histologie } \\
\text { von Screening-Gewebe } \\
\text { (z. B. Aspirat von sub- } \\
\text { kutanem Fett oder Rek- } \\
\text { tumbiopsie) oder Biopsie } \\
\text { von betroffenem Organ. } \\
\text { Kongorotfärbung }\end{array}$ & $\begin{array}{l}\text { Immunhistochemische } \\
\text { Färbung der Gewebs- } \\
\text { biopsie mit Antikör- } \\
\text { pern, welche spezifisch } \\
\text { gegen bestimmte } \\
\text { Amyloid-Fibrillen-Pro- } \\
\text { teine gerichtet sind }\end{array}$ & $\begin{array}{l}\text { Gewebsbiopsie betroffender } \\
\text { Organe; } \\
\text { bei gesicherter Diagnose } \\
\text { werden weitere Organbiop- } \\
\text { sien zur Untersuchung auf } \\
\text { weitere Organbeteiligungen } \\
\text { nicht empfohlen }\end{array}$ & $\begin{array}{l}\text { Knochenmarks-Aspira- } \\
\text { tionszytologie und } \\
\text {-Stanzbiopsie mit Leicht- } \\
\text { ketten-Immunophäno- } \\
\text { typisierung }\end{array}$ & $\begin{array}{l}\text { Follow-up-Biopsien } \\
\text { von betroffenen } \\
\text { Organen oder des } \\
\text { Knochenmarks sind } \\
\text { nicht hilfreich }\end{array}$ \\
\hline $\begin{array}{l}\text { Hämatologie, } \\
\text { Laborchemie, } \\
\text { Immunologie }\end{array}$ & & $\begin{array}{l}\text { Routine-Elektropho- } \\
\text { rese und Immunfixa- } \\
\text { tion aus Serum und } \\
\text { Urin. Quantitative Be- } \\
\text { stimmung des Serum- } \\
\text { spiegels an freien } \\
\text { Leichtketten-Protei- } \\
\text { nen (FLC-Assay) }\end{array}$ & $\begin{array}{l}\text { Harnstoff, Elektrolyte, Krea- } \\
\text { tinin, Albumin, Bestimmung } \\
\text { Eiweiß-Ausscheidung im } \\
\text { 24h-Urin, Lebersynthese- } \\
\text { parameter, Gerinnungslabor, } \\
\text { Kreatinin-Clearance (gemes- } \\
\text { sen oder errechnet) }\end{array}$ & $\begin{array}{l}\text { Großes Blutbild, Harn- } \\
\text { stoff, Elektrolyte inklu- } \\
\text { sive Calcium, Kreatinin, } \\
\text { Albumin. Quantifizie- } \\
\text { rung des Paraproteins in } \\
\text { Serum und Urin. Bestim- } \\
\text { mung der normalen } \\
\text { Immunglobuline. }\end{array}$ & $\begin{array}{l}\text { Paraproteinlevel, } \\
\text { quantitative Be- } \\
\text { stimmung des } \\
\text { Serumspiegels an } \\
\text { freien Leichtketten- } \\
\text { Proteinen } \\
\text { (FLC-Assay) }\end{array}$ \\
\hline Bildgebung & $\begin{array}{l}\text { Serum amyloid P com- } \\
\text { ponent (SAP) Scanning } \\
\text { (Szintigrafie), sofern } \\
\text { verfügbar }\end{array}$ & $\begin{array}{l}\text { Serum amyloid P com- } \\
\text { ponent (SAP) Scanning } \\
\text { (Szintigrafie), sofern } \\
\text { verfügbar }\end{array}$ & $\begin{array}{l}\text { Serum amyloid P component } \\
\text { (SAP) Scanning (Szintigra- } \\
\text { fie), sofern verfügbar }\end{array}$ & Skelett-Diagnostik & $\begin{array}{l}\text { Serum amyloid P } \\
\text { component (SAP) } \\
\text { Scanning (Szintigra- } \\
\text { fie), sofern verfügbar }\end{array}$ \\
\hline Sonstiges & & $\begin{array}{l}\text { DNA-Analyse, Amyloid- } \\
\text { fibrillen-Sequenzierung }\end{array}$ & $\begin{array}{l}\text { EKG, Echokardiografie, } \\
\text { Röntgen-Thorax }\end{array}$ & & $\begin{array}{l}\text { Organ-Funktions- } \\
\text { tests }\end{array}$ \\
\hline
\end{tabular}




\begin{tabular}{|c|c|}
\hline Therapieschema & Indikation \\
\hline \multicolumn{2}{|l|}{ Low dose-Therapie-Ansätze } \\
\hline Melphalan (+ Prednisolon) & $\begin{array}{l}\text { Option, sofern intermediate oder Hochdosistherapie nicht in } \\
\text { Betracht kommt }\end{array}$ \\
\hline \multicolumn{2}{|l|}{ Intermediate dose-Therapie-Ansätze } \\
\hline $\begin{array}{l}\text { Melphalan und Dexamethason } \\
\text { (Palladini-Schema) }\end{array}$ & $\begin{array}{l}\text { Therapie der Wahl, sofern Hochdosistherapie und Stammzelltrans- } \\
\text { plantation nicht durchführbar }\end{array}$ \\
\hline \multicolumn{2}{|l|}{ High dose-Therapie-Ansätze } \\
\hline $\begin{array}{l}\text { High dose-Therapie (HDT) mit Melphalan } \\
\text { und autologe Stammzelltransplantation }\end{array}$ & $\begin{array}{l}\text { Hochdosistherapie und autologe Transplantation wird nicht } \\
\text { empfohlen bei } \\
\text { - symptomatischer Herzamyloidose } \\
\text { - symptomatischer autonomer Neuropathie } \\
\text { - Vorgeschichte von amyloidosebedingter GI-Blutung } \\
\text { - dialysepflichtigem Nierenversagen } \\
\text { - Lebensalter > } 70 \text { Jahre } \\
\text { - > } 2 \text { Organe beteiligt } \\
\text { Autologe Stammzelltransplantation kann außerdem erwogen } \\
\text { werden in selektierten Patienten: } \\
\text { - niedriges Risiko (keine Herzbeteiligung, } 1 \text { - } 2 \text { Organe } \\
\text { betroffen und glomeruläre Filtrationsrate }>50 \mathrm{ml} / \mathrm{min} \text { ) } \\
\text { - frühes Rezidiv der Plasmazelldyskrasie nach Vortherapie }\end{array}$ \\
\hline \multicolumn{2}{|l|}{ Andere Ansätze } \\
\hline $\begin{array}{l}\text { Bortezomib, Cyclophosphamide, } \\
\text { Dexamethason (und autologe } \\
\text { Stammzelltransplantation) }\end{array}$ & $\begin{array}{l}\text { Zu erwägen, falls Hochdosistherapie bzw. Intermediate dose- } \\
\text { Melphalan-Protokoll nicht durchführbar als Erstlinientherapie oder } \\
\text { bei Rezidiv } \\
\text { Möglich auch bei Herzbeteiligung oder Beteiligung von > } 2 \text { Organen }\end{array}$ \\
\hline Bortezomib (+ Dexamethason) & $\begin{array}{l}\text { Zu erwägen, sofern andere Konzepte nicht durchführbar sind auf- } \\
\text { grund von zu erwartender Toxizität oder im Falle von fehlendem } \\
\text { Ansprechen auf Chemotherapie, außerdem bei hohem Spiegel freier } \\
\text { Leichtketten im Serum oder hohem NT-pro-BNP-Spiegel (N-termi- } \\
\text { nales pro-Brain natriuretisches Peptid) } \\
\text { Nicht zu empfehlen bei Amyloidose-bedingter Polyneuropathie }\end{array}$ \\
\hline Lenalidomid (+ Dexamethason) & $\begin{array}{l}\text { Zu erwägen, sofern andere Konzepte nicht durchführbar sind auf- } \\
\text { grund von zu erwartender Toxizität oder im Falle von fehlendem } \\
\text { Ansprechen auf Chemotherapie }\end{array}$ \\
\hline $\begin{array}{l}\text { Intermittierend hochdosiertes } \\
\text { Dexamethason }\end{array}$ & $\begin{array}{l}\text { Zu erwägen, sofern andere Konzepte nicht durchführbar sind auf- } \\
\text { grund von zu erwartender Toxizität oder im Falle von fehlendem } \\
\text { Ansprechen auf Chemotherapie }\end{array}$ \\
\hline Thalidomid (+ Dexamethason) & $\begin{array}{l}\text { Zu erwägen, sofern andere Konzepte nicht durchführbar sind auf- } \\
\text { grund von zu erwartender Toxizität oder im Falle von fehlendem } \\
\text { Ansprechen auf Chemotherapie }\end{array}$ \\
\hline
\end{tabular}

Tab. 3 Therapieoptionen bei generalisierter AL-Amyloidose. Modifiziert nach: Guidelines on the diagnosis and management of AL-Amyloidosis [6].

\section{Amyloidose in der Pneumologie}

Der klinisch tätige Pneumologe wird die Amyloidose eher selten als Ursache für die vom Patienten geschilderten Beschwerden identifizieren. Dennoch ist es essenziell, diese Erkrankung als Differenzialdiagnose bei der Abklärung bestimmter Symptomenkomplexe zu erwägen. Nur so kann der Schritt der wegweisenden Gewebebiopsie mit ins diagnostische Kalkül einbezogen werden, und der Pathologe erhält den entscheidenden Hinweis für die zielführenden Färbemethoden.

Historisch gesehen war die Amyloidose meist eine seltene Zufallsdiagnose, die oft erst durch den befundenden Pathologen gestellt wurde. Zudem war eine rückläufige Prävalenz zu beobachten angesichts der besseren Behandlungsmöglichkeiten einerseits der chronisch entzündlichen Erkrankungen wie Tuberkulose und Bronchiektasen und andererseits der monoklonalen Gammopathien. In den letzten Jahren zeigte sich jedoch, dass bei systematischer Differenzialdiagnostik die Amyloidose weitaus häufiger diagnostiziert wird, als bisher angenommen. Zwar ist die Amyloidose nach wie vor eine seltene Erkrankung, dennoch ist davon auszugehen, dass die Dunkelziffer unverändert hoch ist.
Im Amyloid Register Kiel sind 144 Patienten dokumentiert, bei denen in Biopsaten des Kehlkopfes (30 Fälle), der Trachea (10), Bronchien (28), Lunge (73) und Pleura (3) Amyloid nachgewiesen worden war. Das mittlere Alter der Patienten bei Diagnosestellung betrug 63 Jahre (Umfang 20-85 Jahre; Median 66 Jahre) mit einer deutlichen Männerwendigkeit ( $\mathrm{m}: \mathrm{w}=1,5: 1,0)$. Mit 112 (87\%) Fällen war die AL-Amyloidose (101 Fälle vom lambda- und 11 Fälle vom kappa-Leichtkette-Subtyp) die häufigste Form der Amyloidose der Atemwege und Lunge im Register. Die ATTRAmyloidose wurde mit fünf (3\%) Fällen am zweithäufigsten nachgewiesen. Die AA-Amyloidose fand sich bei vier (3\%) Fällen und die AApoAI-Amyloidose bei einem (0,7\%). In 13 (9\%) Fällen konnte die AL-Amyloidose nicht weiter subklassifiziert werden. Bei neun (6\%) Fällen konnte das Amyloidprotein nicht identifiziert werden. Die aktuelle Registerauswertung zeigt, dass die Amyloidose der Atemwege und Lunge im klinisch-pathologischen Einsendegut (nicht Obduktionsgut) bezüglich der Altersverteilung, Männerwendigkeit und Häufigkeitsverteilung der hepatischen Form nahe kommt. Im Vergleich zu Herz-, Leber- und Nierenbiopsaten wird die AL-Amyloidose vom lambda-Leichtkette-Subtyp am häufigsten in den Atemwegen und der Lunge nachgewiesen ( $\bullet$ Abb. 1, $\odot$ Abb.2). 


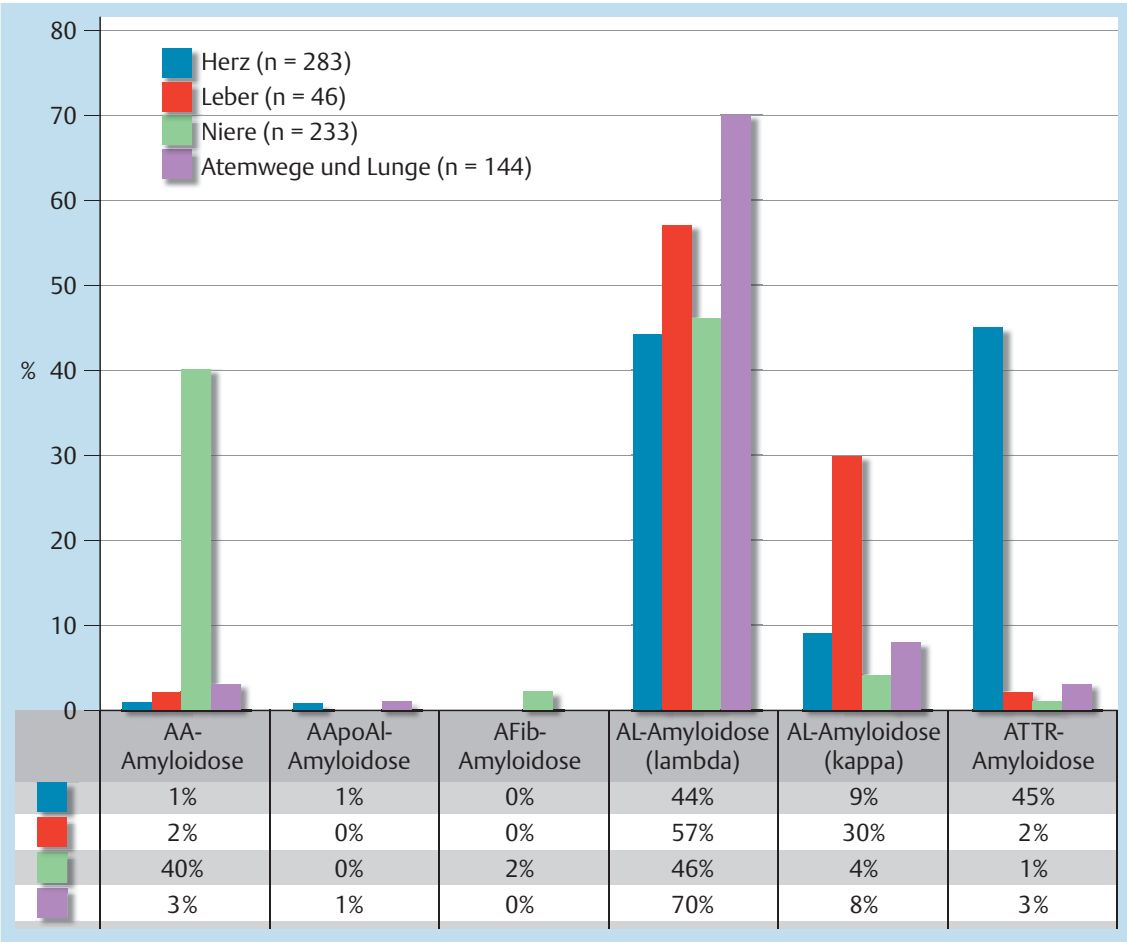

Abb.2 Häufigkeitsverteilung der Amyloidosen in Biopsaten und Resektaten modifiziert nach: Kieninger $B$ et al. [60].

Die Daten des Kieler Amyloidose-Registers korrelieren gut mit älteren epidemiologischen Daten, die insbesondere in den USA erhoben wurden.

Dort konnte bereits an Obduktions- und Operationspräparaten gezeigt werden, dass die AA-Amyloidose sich bei pulmonaler Manifestation überwiegend in den Atemwegen und Pulmonalgefäßen und nur sehr selten im Lungenparenchym im Sinne einer alveoloseptalen Amyloidose manifestiert [18-20].

Eine gewisse Sonderstellung stellten AA-Amyloidosen dar, die mit dem familiären Mittelmeerfieber assoziiert waren. Selten wurde bei dieser Form eine Lungenparenchym-Beteiligung, eine mediastinale Lymphadenopathie oder pulmonale Hypertonie nachgewiesen [20-22].

Die TTR-assoziierte Amyloidose wurde in historischen Kollektiven insgesamt sehr selten nachgewiesen, zeigte jedoch in verschiedenen Untersuchungen relativ häufig eine Manifestation im Lungenparenchym. Bei den senilen TTR-Amyloidosen lag die Häufigkeit altersabhängig zwischen $2 \%$ und $20 \%[9,19,23,24]$.

Bei Patienten mit einer generalisierten AL-Amyloidose zeigten sich in der systematischen Aufarbeitung von Autopsiepräparaten regelhaft auch Amyloidablagerungen in den Atemwegen, den pulmonalen Gefäßen und dem Lungenparenchym [18]. Es konnte gezeigt werden, dass die Lungenbeteiligung jedoch nur bei wenigen Patienten im Verlauf der Erkrankung zu Symptomen führt. Nur in 1/12 der Fälle war die Lungenbeteiligung ausschlaggebend für den klinischen Verlauf, nur in etwa 10\% führte die Lungenbeteiligung zum Tod [25].

Historische Daten zeigten außerdem, dass bei pulmonaler Manifestation einer generalisierten AL-Amyloidose in der Regel auch eine Herzbeteiligung vorliegt, die dann meist auch prognosebestimmend war [26].

Gerade in der Pneumologie manifestiert sich die Amyloidose mit einem sehr heterogenen Spektrum an Krankheitsbildern. Es können hereditäre wie erworbene Amyloidosen vorliegen, je nach Erkrankungsbild dominieren systemische oder lokale Formen. Im Bereich des Respirationstraktes kommen AA-Amyloidosen zwar vor, werden jedoch bedeutend seltener beobachtet als die AL-Amyloidosen [16, 27 -29]. Insbesondere bei den generalisierten Formen der AA-Amyloidose und der AL-Amyloidose steht die kausale Behandlung der Grunderkrankung und damit eine systemische Therapie im Vordergrund. Bei den lokalen Amyloidoseformen werden in der Regel lokale Behandlungsmaßnahmen mit gutem Erfolg und anhaltendem Benefit durchgeführt. Ein diagnostischer und therapeutischer Algorithmus kann helfen, mithilfe einer rationalen Umgebungsdiagnostik Organbeteiligungen zu identifizieren, die Indikation für eine spezifische systemische Therapie zu stellen und Verläufe zu beurteilen ( $\bullet$ Abb. 3 und - Abb.4). Aufgrund der Komplexität des Krankheitsbildes und der mannigfaltigen Erscheinungsformen muss jedoch häufig eine individualisierte Behandlungsplanung erfolgen.

\section{Schlafbezogene Atemstörungen}

Amyloid-Ablagerungen im Bereich der oberen Atemwege, typischerweise in Zunge und Rachen, können schwere schlafbezogene Atemstörungen zur Folge haben. Ursächlich sind in der Literatur sowohl erworbene als auch hereditäre Amyloidoseformen beschrieben [30,31], wobei die Makroglossie jedoch pathognomonisch für die AL-Amyloidose ist. In der Polysomnografie kann dabei nicht selten eine obstruktive Schlafapnoe diagnostiziert werden. Vor Einführung der CPAP-Therapie (Continuous Positive Airway Pressure) war die Tracheotomie die einzige Behandlungsoption [32]. Heute werden die CPAP-Therapie und die nichtinvasive Beatmung mit Erfolg eingesetzt.

\section{Stridor}

Im Kehlkopfbereich können Amyloidablagerungen zu Heiserkeit und Stridorsymptomatik durch laryngeale Stenosen oder Stimmbandparesen führen. Hier dominieren lokale Formen der ALAmyloidose und die hereditäre AApoAI-Amyloidose. Seit Einführung der interventionellen Lasertherapie kann in den meisten Fällen eine Tracheotomie vermieden werden. Als weiteres Standardverfahren wird das Debulking durch endoskopische Exzision 


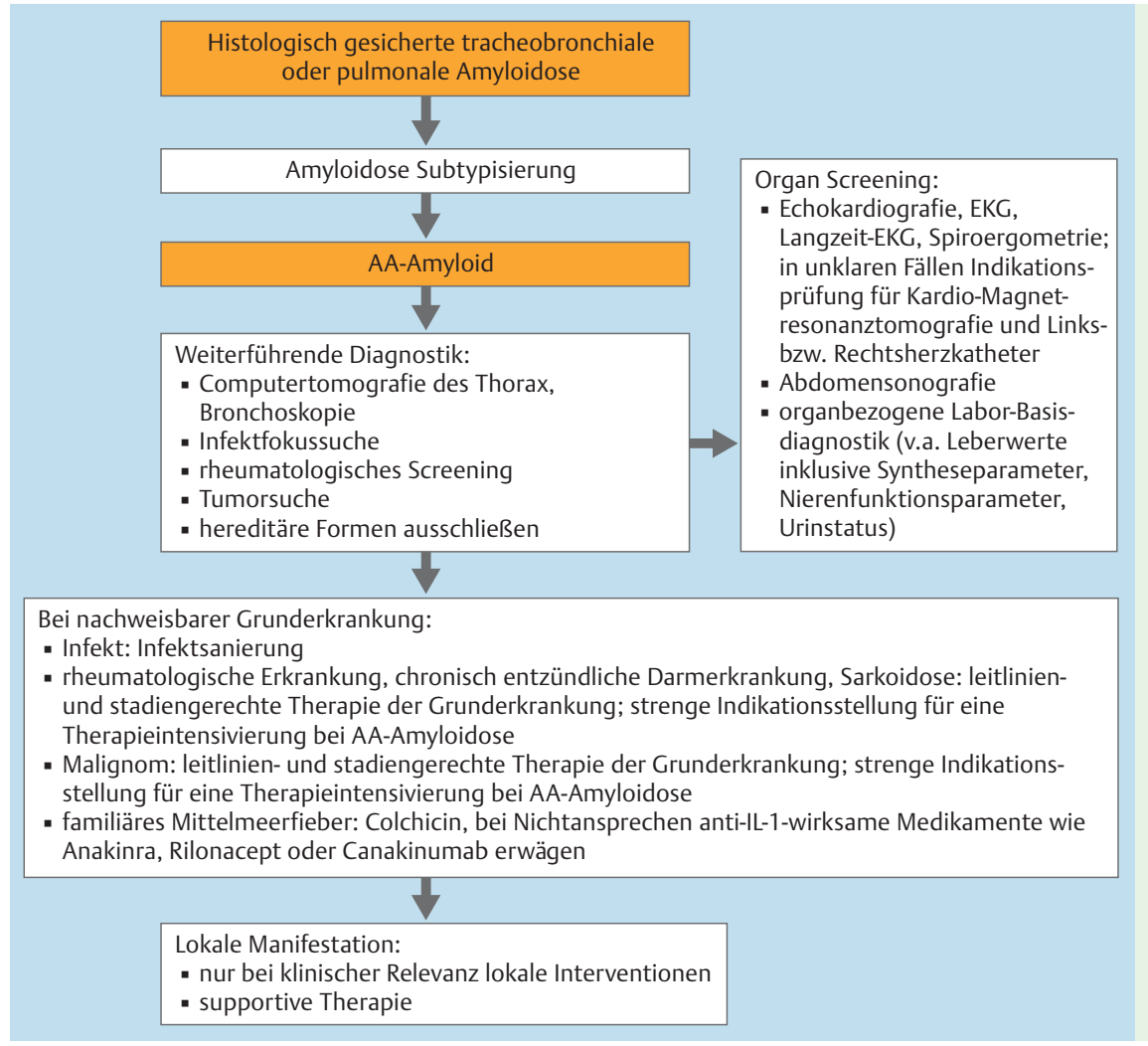

Abb.3 Algorithmus zur Diagnostik und Therapie bei gesicherter AA-Amyloidose.

Bei nachweisbarer Grunderkrankung:

- Infekt: Infektsanierung

und stadiengerechte Therapie der Grunderkrankung; strenge Indikationsstellung für eine

pintensivierung bei AA-Amyloidose

- nur bei klinischer Relevanz lokale Interventionen

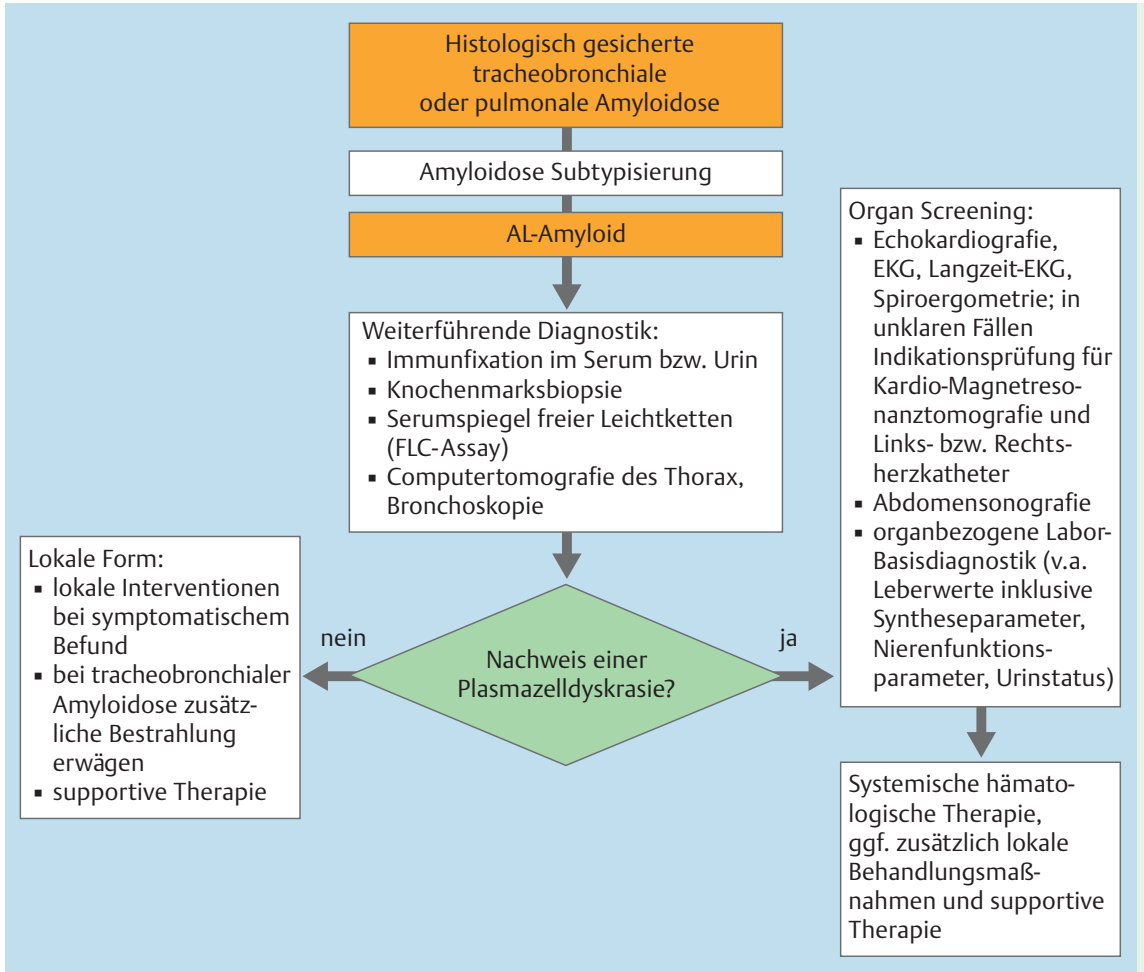

Abb.4 Algorithmus zur Diagnostik und Therapie bei gesicherter AL-Amyloidose.

angesehen. Möglicherweise kann durch eine ergänzende Bestrahlung die Rezidivneigung reduziert werden [33,34].

Die tracheobronchiale Amyloidose wird ebenso wie die laryngeale Form meist durch eine lokale AL-Amyloidose verursacht. Im Vordergrund des Therapiekonzepts stehen in diesen Fällen lokale Behandlungsmaßnahmen. Ablagerungen im Bereich der zentralen Atemwege oder der mediastinalen Lymphknoten können zu relevanten zentralen Atemwegsobstruktionen führen. Kompressionsbedingte kurzstreckige Stenosen können mit gutem Erfolg durch eine tracheale oder bronchiale Stentimplantation behandelt werden. Endobronchiale Amyloidome, die wie stenosierende Tumore imponieren können, werden durch Rekanalisationsmaßnahmen wie z.B. Laserintervention oder Zangenexzision mit gutem Erfolg abgetragen. Lokalrezidive treten selten auf. Lang- 

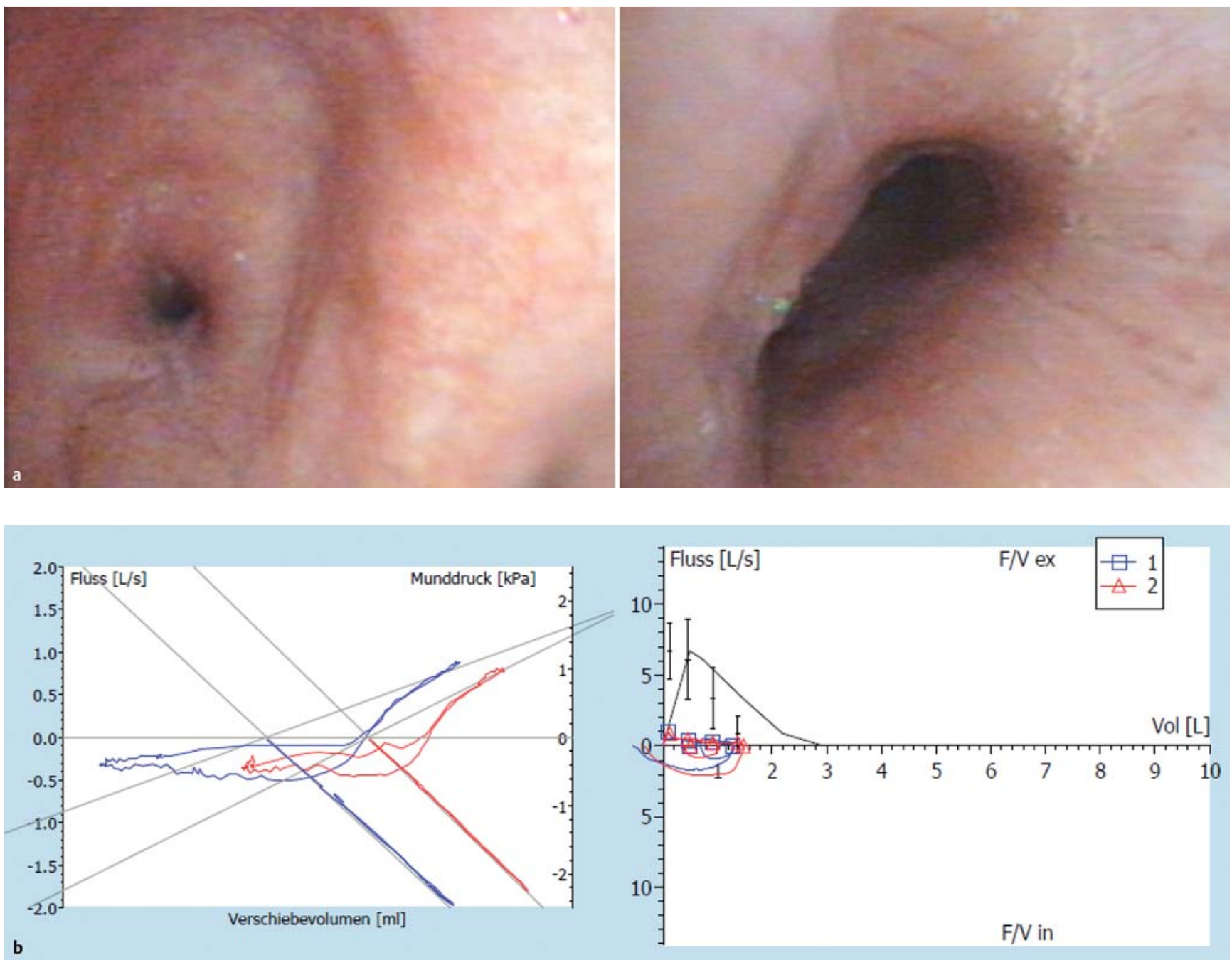

Abb.5 Bronchoskopiebefund einer hochgradigen langstreckigen tracheobronchialen Stenosierung bei diffuser tracheobronchialer Amyloidose, links: Eingang Trachea, rechts: linker Hauptbronchus (a). Lungenfunktionell liegt bei diesem Fall der Befund einer schwerstgradigen zentralen und peripheren Obstruktion der Atemwege vor bei einem massiv erhöhten Atemwegswiderstand von R eff 2,23 kPa*s/L(742\%Soll), SR eff 10,11 kPa*s (860\%Soll) und eingeschränktem Tiffeneau-Index FEV1/IVC 26,4\% bei einem Atemstoßwert von FEV1 0,48I (22\%Soll). Formal findet sich außerdem der Befund einer leichtgradigen Überblähung sowie einer leicht- bis mittelgradigen Restriktion. Blutgasanalytisch liegt eine gemischt respiratorische und metabolische Azidose vor mit einem pH von 7,28. Es findet sich eine relevante Hypoxie (pO2 53,9 mm Hg) und Hyperkapnie (pCO2 51,9 mmHg) als Hinweis auf ein akut drohendes Atemversagen (b).

streckige amyloidbedingte Stenosierungen können diffus das gesamte Tracheobronchialsystem bis auf Segmentebene betreffen ( Abb.5). Diese Form ist selten, die Prognose ist schlecht aufgrund der bei generalisiertem tracheobronchialem Befall letztlich fehlenden interventionellen Therapieoptionen. Dilatationen, Stents oder Laserinterventionen haben bei diesen Patienten in der Regel allenfalls einen kurzfristigen Effekt.

\section{Ventilatorische Insuffizienz}

Eine ventilatorische Insuffizienz kann in seltenen Fällen bedingt sein durch eine Amyloidneuropathie oder eine Amyloidmyopathie mit Beteiligung von Zwerchfell bzw. Atemhilfsmuskulatur [35-38]. Diese Manifestationsformen wurden sowohl für erworbene als auch für angeborene Amyloidosen beschrieben $[39,40]$. Sofern vertretbar sollte in der Behandlung der nichtinvasiven vor der invasiven Beatmung der Vorzug gegeben werden.

\section{Gasaustauschstörung}

Die diffuse alveolar-septale Amyloidose ist fast immer eine ALAmyloidose und tritt als primäre lokale Amyloidose auf oder generalisiert als sekundäre Manifestation bei einer gesicherten monoklonalen Gammopathie. Bei der generalisierten Form finden sich in aller Regel noch andere Organmanifestationen. In der Literatur sind lediglich einzelne Kasuistiken beschrieben mit dem Bild einer alveoloseptal betonten interstitiellen Lungenamyloidose auf dem Boden einer AA-Amyloidose [28,41].

In den meisten Fällen führt das Symptom einer unklaren Belastungsdyspnoe zur erweiterten Diagnostik mittels Röntgen und hochauflösender Computertomografie des Thorax (HR-CT). Es imponiert das Bild einer interstitiellen Zeichnungsvermehrung mit septaler Betonung und überwiegend retikulärem Muster. Die CT-Morphologie bleibt in der Regel unspezifisch und muss zur erweiterten Abklärung führen. Die klare pathologische Diagnosestellung wird häufig erst mit der chirurgischen Lungenbiopsie möglich, seltener sind bronchoskopisch gewonnene transbronchiale Lungenbiopsien ausreichend [28,42]. 
In der Behandlung kann eine systemische Chemotherapie die insgesamt schlechte Prognose in der Regel kaum beeinflussen. Bei fortgeschrittenen Stadien wird eine Sauerstoffverordnung erforderlich. Die Lungentransplantation kann als Ultima Ratio durchgeführt werden, sofern dies unter Berücksichtigung anderer Organmanifestationen vertretbar erscheint [43].

\section{Pulmonale Rundherde}

Die noduläre Amyloidose ist fast immer eine Zufallsdiagnose bei der Abklärung pulmonaler Rundherde bzw. Raumforderungen. Häufig bleibt dabei die Bronchoskopie ohne wegweisenden Befund, erst am chirurgischen Resektat lässt sich dann die Diagnose stellen. Die noduläre Amyloidose kann durch AA-Amyloid oder durch AL-Amyloid bedingt sein [29]. In der Literatur ist außerdem der Fall einer pulmonalen nodulären Amyloidose beschrieben, bei der letztlich eine erworbene senile Amyloidose-Form mit ATTR-Amyloid als Vorläuferprotein nachgewiesen wurde [44]. Die tumorförmigen Amyloidosen der Lunge können außerdem heterotope Ossifikationen aufweisen, die u.U. bildmorphologisch auffällig sind ( $\bullet$ Abb. 1$)$.

Bei großen raumfordernden bzw. komplizierten Amyloidomen ist die Operation die Therapie der Wahl, Rezidive sind selten [45]. Bei disseminierten Rundherden ist nach Diagnosestellung aufgrund der guten Prognose keine radikale Operation im Sinne von multiplen Keilresektionen erforderlich [46, 47]. Spontanremissionen sind in der Literatur auch für AA-Amyloidome beschrieben [27].

\section{Pulmonale Hypertonie}

Die differenzialdiagnostische Abklärung einer pulmonalen Hypertonie kann zum Befund einer kardialen systolischen oder diastolischen Funktionsstörung, bedingt durch eine Herzamyloidose, führen. Die Prognose ist in diesen Fällen sehr schlecht. Die Indikation für eine Herz-Transplantation sollte interdisziplinär unter besonderer Berücksichtigung der Gesamtkonstellation geprüft werden.

In der Literatur sind bei Amyloidosepatienten auch Fälle einer pulmonalen Hypertonie ohne Hinweis für eine Herzamyloidose beschrieben [21,48-51]. Eine ausreichend evaluierte Behandlung steht für diese Patienten nicht zur Verfügung. Die spezifischen PAH-Medikamente sind für diese Indikation nicht zugelassen. Eine Lungentransplantation oder gegebenenfalls Herz-Lungen-Transplantation kann unter den oben genannten Voraussetzungen eine Option darstellen.

\section{Hämoptysen}

Unklare Hämoptysen können in seltenen Fällen zur Diagnose einer Amyloidose führen. Die Blutungen können verursacht sein durch eingeblutete noduläre Amyloidome oder durch eine pulmonale Gefäßamyloidose mit komplizierender alveolärer Hämorrhagie [52]. Blutende noduläre Amyloidome können mit gutem Langzeiterfolg operativ entfernt werden. Bei den einzelnen beschriebenen Fällen einer pulmonalen Gefäßamyloidose wurde eine kurzfristige hochdosierte Steroidtherapie zur Behandlung der alveolären Hämorrhagie mit Erfolg durchgeführt [53].

\section{Lungeninfarkt}

In seltenen Fällen ist ein Lungeninfarkt durch eine pulmonale Amyloidangiopathie bedingt. Die Diagnosestellung erfolgt entweder im Rahmen einer diagnostischen Thorakotomie zur Abklärung eines unklaren Infiltrates oder post mortem in der Obduktion [54].
Pleuraergüsse und pleurale Raumforderungen

Persistierende bzw. rezidivierende Pleuraergüsse treten bei 1-2\% aller Patienten mit einer generalisierten Amyloidose auf [55]. Beschrieben sind Manifestationen im Rahmen einer AL-, einer AAAmyloidose sowie einer Dialyse-assoziierten Amyloidose (ß2Mikroglobulin als Amyloid-Vorläuferprotein) [56]. Die Behandlung der Grunderkrankung kann hier bereits zu einer deutlichen Befundbesserung beitragen $[57,58]$. Teils gehen die Pleuraergüsse mit Verschwartungen und Verkalkungen an der Pleura einher, die ebenfalls als Amyloidosemanifestationen zu werten sind. Bei therapierefraktären Ergüssen kann eine thorakoskopische Talkumpoudrage durchgeführt werden [59].

\section{Amyloidose-Netzwerke und Fachgesellschaften $\nabla$}

Es sei an dieser Stelle auf Netzwerke internationaler Amyloidoseverbände und der deutschen Amyloidosegesellschaft verwiesen. Deutsche Amyloidosegesellschaft www.amyloid.de International Society of Amyloidosis (ISA) www.amyloidosis.nl Peter Waldmann Amyloidose-Stiftung www.amyloidose.ch

\section{Kontaktdaten Selbsthilfegruppen}

Arbeitsgemeinschaft Plasmozytom/MultiplesMyelom (APMM) www.myelom.org

Private Homepage und Selbsthilfegruppe für Patienten mit familiärem Mittelmeerfieber www.mittelmeerfieber.de

\section{Interessenkonflikt}

Die Autoren geben an, dass kein Interessenkonflikt besteht.

\section{Literatur}

1 Sipe JD, Cohen AS. Review: history of the amyloid fibril. J Struct Biol 2000; 130: 88-98

2 Schonland SO, Moos M, Bochtler T et al. [Systemic light chain amyloidosis - molecular basis and clinical perspectives]. Deutsche medizinische Wochenschrift 2009; 134: 1949-1952

3 Schonland SO, Bochtler T, Kristen AV et al. [Current diagnostic and therapy of light chain amyloidosis]. Pathologe 2009; 30: 205-211

4 Libbey CA, Skinner M, Cohen AS. Use of abdominal fat tissue aspirate in the diagnosis of systemic amyloidosis. Archives of internal medicine 1983; 143: 1549-1552

5 Duston MA, Skinner M, Shirahama T et al. Diagnosis of amyloidosis by abdominal fat aspiration. Analysis of four years' experience. The American journal of medicine 1987; 82: 412-414

6 Guidelines on the diagnosis and management of $\mathrm{AL}$ amyloidosis. $\mathrm{Br}$ Haematol 2004; 125: $681-700$

7 Kyle RA, Gertz MA. Primary systemic amyloidosis: clinical and laboratory features in 474 cases. Semin Hematol 1995; 32: 45 - 59

8 Cohen AD, Comenzo RL. Systemic light-chain amyloidosis: advances in diagnosis, prognosis, and therapy. Hematology Am Soc Hematol Educ Program 2010; 2010: 287-294

9 Sipe JD, Benson MD, Buxbaum JN et al. Amyloid fibril protein nomenclature: 2010 recommendations from the nomenclature committee of the International Society of Amyloidosis. Amyloid 2010; 17: 101 - 104

10 Rocken C, Ernst J, Hund E et al. Interdisciplinary guidelines for diagnosis and therapy of extracerebral amyloidosis: issued by the German Society of Amyloid Diseases e.V. Medizinische Klinik 2006; 101: 825 - 829

11 Eriksson M, Buttner J, Todorov $T$ et al. Prevalence of germline mutations in the TTR gene in a consecutive series of surgical pathology specimens with ATTR amyloid. The American journal of surgical pathology 2009; 33: $58-65$

12 Kyle RA, Therneau TM, Rajkumar SV et al. A long-term study of prognosis in monoclonal gammopathy of undetermined significance. $\mathrm{N}$ Engl J Med 2002; 346: 564-569 
13 Mikhael JR, Schuster SR, Jimenez-Zepeda VH et al. Cyclophosphamidebortezomib-dexamethasone (CYBORD) produces rapid and complete hematological response in patients with AL amyloidosis. Blood 2012: Epub ahead of print

14 Venner CP, Lane T, Foard D et al. Cyclophosphamide, bortezomib and dexamethasone therapy in AL amyloidosis is associated with high clonal response rates and prolonged progression free survival. Blood 2012: Epub ahead of print

15 Akcay S, Akman B, Ozdemir $H$ et al. Bronchiectasis-related amyloidosis as a cause of chronic renal failure. Renal failure 2002; 24: 815-823

16 Levasseur R, Le Goff C, Richer C et al. [AA amyloidosis complicating sarcoidosis]. Rev Med Interne 1999; 20: 168-170

17 Yonem 0, Bayraktar Y. Secondary amyloidosis due to FMF. Hepatogastroenterology 2007; 54: 1061 - 1065

18 Celli BR, Rubinow A, Cohen AS et al. Patterns of pulmonary involvement in systemic amyloidosis. Chest 1978; 74: 543-547

19 Smith RR, Hutchins GM, Moore GW et al. Type and distribution of pulmonary parenchymal and vascular amyloid. Correlation with cardiac amyloid. The American journal of medicine 1979; 66: 96-104

20 Utz JP, Swensen SJ, Gertz MA. Pulmonary amyloidosis. The Mayo Clinic experience from 1980 to 1993. Annals of Internal medicine 1996; 124 : $407-413$

21 Johnson WJ, Lie JT. Pulmonary hypertension and familial Mediterranean fever: a previously unrecognized association. Mayo Clinic proceedings. Mayo Clinic 1991; 66: 919-925

22 Livneh A, Langevitz P, Pras M. Pulmonary associations in familial Mediterranean fever. Curr Opin Pulm Med 1999; 5: 326 - 331

23 Kunze WP. Senile pulmonary amyloidosis. Pathol Res Pract 1979; 164 : 413-422

24 Pitkanen P, Westermark P, Cornwell GG 3rd et al. Senile systemic amyloidosis. The American journal of pathology 1984; 117: 391 - 399

25 Cordier JF, Loire R, Brune J. Amyloidosis of the lower respiratory tract. Clinical and pathologic features in a series of 21 patients. Chest 1986; 90: $827-831$

26 Berk JL, O'Regan A, Skinner M. Pulmonary and tracheobronchial amyloidosis. Semin Respir Crit Care Med 2002; 23: 155-165

27 Fukatsu H, Miyoshi H, Ishiki K. Spontaneous resolution of multiple nodular pulmonary AA amyloidosis. Internal medicine 2010; 49: 2303 2307

28 Planes C, Kleinknecht D, Brauner $M$ et al. Diffuse interstitial lung disease due to AA amyloidosis. Thorax 1992; 47: 323 - 324

29 Calatayud J, Candelas G, Gomez A et al. Nodular pulmonary amyloidosis in a patient with rheumatoid arthritis. Clinical rheumatology 2007; 26: $1797-1798$

30 Kiuru S, Nieminen T, Partinen M. Obstructive sleep apnoea syndrome in hereditary gelsolin-related amyloidosis. Journal of sleep research 1999; 8: 143 - 149

31 Lesser BA, Leeper KV Jr, Conway W. Obstructive sleep apnea in amyloidosis treated with nasal continuous positive airway pressure. Archives of internal medicine 1988; 148: 2285-2287

32 Carbone JE, Barker D, Stauffer JL. Sleep apnea in amyloidosis. Chest 1985; 87: $401-403$

33 Gallivan GJ, Gallivan HK. Laryngeal amyloidosis causing hoarseness and airway obstruction. J Voice 2010; 24: 235-239

34 Neuner GA, Badros AA, Meyer TK et al. Complete resolution of laryngeal amyloidosis with radiation treatment. Head Neck 2012; 34: $748-752$

35 Ashe J, Borel CO, Hart G et al. Amyloid myopathy presenting with respiratory failure. Journal of neurology, neurosurgery, and psychiatry 1992; 55: $162-165$

36 Berk JL, Wiesman JF, Skinner $M$ et al. Diaphragm paralysis in primary systemic amyloidosis. Amyloid 2005; 12: 193 - 196

37 Santiago RM, Scharnhorst D, Ratkin $G$ et al. Respiratory muscle weakness and ventilatory failure in AL amyloidosis with muscular pseudohypertrophy. The American journal of medicine 1987; 83: 175-178
38 Streeten EA, de la Monte SM, Kennedy TP. Amyloid infiltration of the diaphragm as a cause of respiratory failure. Chest 1986; 89: $760-762$

39 Gagnon RF, Thirlweil M, Arzoumanian A et al. Systemic amyloidosis involving the diaphragm and acute massive hydrothorax during peritoneal dialysis. Clinical nephrology 2002; 57: 474-479

40 Liu JY, Guo YJ, Zhou CK et al. Clinical and histopathological features of familial amyloidotic polyneuropathy with transthyretin Val30Ala in a Chinese family. Journal of the neurological sciences 2011; 304: 83 - 86

41 Erdem H, Simsek I, Pay S et al. Diffuse pulmonary amyloidosis that mimics interstitial lung disease in a patient with familial Mediterranean fever. J Clin Rheumatol 2006; 12: 34-36

42 Konietzko N. Pulmonary amyloidosis. Deutsche Medizinische Wochenschrift 2001; 126: 1369-1372

43 Ware LB, Keith FM, Gordon RL et al. Lung transplantation for pulmonary amyloidosis: a case report. J Heart Lung Transplant 1998; 17: 1129 1132

44 Roden AC, Aubry MC, Zhang K et al. Nodular senile pulmonary amyloidosis: a unique case confirmed by immunohistochemistry, mass spectrometry, and genetic study. Human pathology 2010; 41: 1040-1045

45 Rosen DJ, Stavropoulos C, Travis WD et al. Transdiaphragmatic amyloidoma. Ann Thorac Surg 2008; 86: 310-312

46 Gaurav K, Panda M. An uncommon cause of bilateral pulmonary nodules in a long-term smoker. J Gen Intern Med 2007; 22: 1617-1620

47 Gillmore JD, Hawkins PN. Amyloidosis and the respiratory tract. Thorax 1999; 54: 444-451

48 Chapman AD, Brown PA, Kerr KM. Right heart failure as the dominant clinical picture in a case of primary amyloidosis affecting the pulmonary vasculature. Scott Med J 1999; 44: 116-117

49 Dingli D, Utz JP, Gertz MA. Pulmonary hypertension in patients with amyloidosis. Chest 2001; 120: 1735-1738

50 Eder L, Zisman D, Wolf $R$ et al. Pulmonary hypertension and amyloidosis - an uncommon association: a case report and review of the literature. J Gen Intern Med 2007; 22: 416-419

51 Shiue ST, McNally DP. Pulmonary hypertension from prominent vascular involvement in diffuse amyloidosis. Archives of internal medicine 1988; 148: $687-689$

52 Sterlacci $W$, Veits $L$, Moser $P$ et al. Idiopathic systemic amyloidosis primarily affecting the lungs with fatal pulmonary haemorrhage due to vascular involvement. Pathol Oncol Res 2009; 15: 133-136

53 Shenin $M$, Xiong $W$, Naik $M$ et al. Primary amyloidosis causing diffuse alveolar hemorrhage. J Clin Rheumatol 2010; 16: 175-177

54 Parambil JG, Savci CD, Tazelaar HD et al. Causes and presenting features of pulmonary infarctions in 43 cases identified by surgical lung biopsy. Chest 2005; 127: $1178-1183$

55 Mansalis KA, Klein DA, Demartini SD et al. Pleural findings in a patient with persistent pulmonary effusions from systemic amyloidosis. Amyloid 2011; 18: 29-31

56 Suzuki M, Betsuyaku T, Kojima T et al. Pleural involvement of dialysisrelated amyloidosis. Internal medicine 2005; 44: 628-631

57 Araoka $T$, Takeoka $H$, Nishioka $K$ et al. Successful management of refractory pleural effusion due to systemic immunoglobulin light chain amyloidosis by vincristine adriamycin dexamethasone chemotherapy: a case report. Journal of medical case reports 2010; 4: 322

58 Wirtz G, Andres E, Kessler R. [Thoracic impairment from familial Mediterranean fever: review of the literature and a case study]. Revue de pneumologie clinique 2009; 65: $313-317$

59 Leleu 0 , Clarot C, Renaud $H$ et al. [Amyloidosis and pleural calcification]. Revue des maladies respiratoires 2003; 20: 618-621

60 Kieninger B, Eriksson $M$, Kandolf $R$ et al. Amyloid in endomyocardial biopsies. Virchows Archiv : an international journal of pathology 2010; 456: $523-532$ 